\title{
Anticoncepción con implantes subdérmicos (Norplant) en mujeres adolescentes: Observaciones preliminares en 174 casos
}

\author{
Rodrigo Cifuentes; Agustín Conde A; Luz Angela Torres*
}

\begin{abstract}
RESUMEN: Se presentan resultados de 174 mujeres adolescentes, usuarias de primera vez del Norplant. Se encontró que la edad promedio fue 17,7 años; $39.9 \%$ eran nulíparas. La tasa acumulada de embarazos a 2 años fue de $0 \%$. El $48 \%$ tuvieron ciclos irregulares (9\% manchado, $20 \%$ sangrado y $19 \%$ amenorrea). Otros efectos adversos reportados fueron: mareos $(6 \%)$, cefalea (4\%) y aumento de peso (4\%). La tasa de retiro fue de $6.8 \%$. Las restantes usuarias están satisfechas con el método.

Los anteriores datos nos permiten sugerir el uso del Norplant como método contraceptivo en este grupo etario. Sin embargo, un estudio prolongado ( 5 años), es necesario para corroborar estos hallazgos.

PALABRAS CLAVES: Norplant, adolescentes, contracepción.

SUMMARY: The results of 174 adolescent women, new users of Norplant are presented. The mean age was 17.7 years and $39.9 \%$ were nulliparous. The cumulative rates of pregnancies at the end of two year follow-up was $0 \%$. Forty-eight percent of the users had irregularities of the menstrual cycle (spotting $9 \%$, bleeding $20 \%$, and amenorrhea $19 \%)$. Other adverse effects informed were: dizziness $(60 \%)$, headache $(4 \%)$, and weight gain $(4 \%)$. The retirement rate of the implant was $6.8 \%$. The rest of the users are satisfied with the method.
\end{abstract}

With the previous data we suggest the use on Norplant as a contraceptive method in the group of adolescents. However it is necessary a longer follow-up ( 5 years) to confirm these findings.

KEY WORDS: Norplant, adolescents, contraception.

\section{Introducción}

A medida que la población adolescente inicia su vida sexual activa demanda atención contraceptiva en busca de métodos eficaces, seguros, cómodos y reversibles de acción prolongada. Dentro de estos métodos se encuentra el Implante Subdérmico NORPLANT, el cual proporciona protección eficaz por un período de 5 años. Consiste de 6 cápsulas cilíndricas de silastic, que contiene cada una $36 \mathrm{mg}$ de Levonorgestrel y miden $34 \mathrm{~mm}$ de longitud y $2.4 \mathrm{~mm}$ de diámetro. Estas son insertadas bajo la piel de la cara interna del brazo desde donde el esteroide es liberado inmediatamente a una razón que declina hasta hacerse constante alrededor de los 6-8 meses de 0.6-0.8 $\mathrm{ng} / 24$ horas a $0,3-0,5 \mathrm{ng} /$ día (1-2).

El Norplant actúa por varios mecanismos: 1. Por supresión parcial de la liberación hipofisiaria de FSH y LH. 2. Produciendo aumento de la viscosidad y espesamiento del moco cervical, con la consecuente inhibición de la penetración de los espermatozoides, siendo éste el mecanismo contraceptivo más importante. 3. Por último, las alteraciones a nivel endometrial que consisten en supresión parcial del desarrollo o maduración asincrónica de las glándulas y del estroma (2-4).

El efecto colateral más frecuente en las usuarias del Norplant son alteraciones en el patrón del ciclo menstrual y cefalea.

La eficacia contraceptiva del Norplant no es influida por la disminución de su efectividad en pacientes con más de $70 \mathrm{Kg}$ de peso (5-6).

Asociación Pro-Bienestar de la Familia Colombiana. Profamilia Cali - Colombia.
La tasa acumulada de embarazos al final de los 5 años es de 3.9 por 100 usuarias continuas y la incidencia de embarazos ectópicos es de 0.28/1000 años-mujer, la cual es mucho más baja que en población general $(1,5 / 1000$ años-mujer) (2).

Tomando como referencia estos datos obtenidos a lo largo de 25 años de estudio, quisimos evaluar la aceptabilidad, tolerancia y efectividad del Norplant en la población adolescente.

\section{Pacientes y métodos}

Se realizó un estudio clínico en mujeres adolescentes que solicitaron espontáneamente un método anticonceptivo. Previa evaluación médica para descartar entidades médicas que pudieran contraindicar el uso del levonorgestrel como anticonceptivo, se citó a la usuaria en los primeros 8 días del ciclo menstrual para insertar el implante subdérmico. Una vez colocado el Norplant, de acuerdo a la técnica convencional, se controló a la usuaria en los meses $1,3,6,12,18$ y 24 postinserción.

Durante cada control se registraron datos clínicos de relevancia, entre otros la tensión arterial, el peso, los trastornos del ciclo menstrual, posibles efectos colaterales relacionados con el método, así como niveles de satisfacción y, en caso de solicitar el retiro de los implantes, el motivo para ello.

\section{Resultados}

Hasta la fecha se han aplicado Implantes Subdérmicos a 365 adolescentes, de las cuales 174 han completado 2 años de seguimiento. 
Las características sociodemográficas se muestran en la tabla 1. Para descartar, la mayoría de nuestras usuarias (el $87 \%$ ) se encuentran en la fase final de su adolescencia (entre 17 y 19 años), la mitad de ellas vivían en unión libre $(48,9 \%)$ y al menos habían tenido 1 hijo $(55,7 \%)$.

Los efectos colaterales se observan en la tabla 2 , siendo los más frecuentemente observados las irregularidades en el ciclo menstrual (en el 47,9\% de las usuarias); éstas se presentaron al menos una vez durante el período de seguimiento de 2 años y consistieron en manchado o pintas $(9,3 \%)$, sangrado $(21,2 \%)$ y amenorrea $(19,3 \%)$. Otros efectos secundarios de menor frecuencia fueron mareos $(6,4 \%)$ cefalea $(4,3 \%)$, variaciones en el peso $(4,3 \%)$ acné $(2 \%)$ y caída del cabello $(1,4 \%)$.

En las 174 adolescentes con Norplant, seguidas hasta los 2 años, se presentaron 12 retiros (el 6,8\%), cuyas causas fueron: alteraciones del ciclo menstrual $(1,7 \%)$, deseo de embarazo $(2,3 \%)$, cefalea $(1,7 \%)$ y cambio de peso $(1,1 \%)$, el resto de las usuarias se mostraron satisfechas con el método (92\%).

No se presentó ningún embarazo con el Norplant durante este período.

\section{Conclusiones}

En el presente estudio podemos destacar cómo la mitad de nuestras adolescentes están llegando al final de esta etapa

$$
\text { Tabla } 1
$$

\section{CARACTERISTICAS SOCIODEMOGRAFICAS}

\begin{tabular}{|crr|}
\hline EDAD (Años) & $\mathrm{n}$ & $\%$ \\
$14-17$ & 28 & 14.1 \\
$17-19$ & 146 & 86,9 \\
ESCOLARIDAD & $\mathrm{n}$ & $\%$ \\
Bachillerato & 160 & 92,0 \\
Universitario & 14 & 8,0 \\
ESTADO CIVIL & $\mathrm{n}$ & $\%$ \\
Soltera & 36 & 20,7 \\
Casada & 53 & 30,5 \\
Unión Libre & 85 & 48,9 \\
PARIDAD & $\mathrm{n}$ & $\%$ \\
0 & 67 & 38,5 \\
1 & 97 & 55.7 \\
2 & 10 & 5,7 \\
ABORTOS & $\mathrm{n}$ & $\%$ \\
0 & 149 & 85,6 \\
1 & 23 & 13,2 \\
2 & 2 & 1,1 \\
PESO (Kg) & $\mathrm{n}$ & $\%$ \\
$40-50$ & 50 & 28,7 \\
$51-60$ & 96 & 55,2 \\
$61-70$ & 28 & 16,1 \\
\hline
\end{tabular}

apenas culminando sus estudios básicos -el 85,9\% están entre los 17 y 19 años, y el $92 \%$ están cursando bachillerato- con la responsabilidad temprana de un hogar - son casadas el 30,6\%; en unión libre el 48,9\% - o están enfrentadas al madresolterismo (el $36 \%$ de las usuarias) y más de la mitad de ellas al menos tienen un hijo (el 55,7\%).

Se hace entonces necesaria la oferta de métodos anticonceptivos confiables que permitan a las adolescentes ejercer su derecho de elegir en lo relativo a su propia fecundidad.

El Norplant se constituye en una eficaz, segura, cómoda y prolongada opción dada su alta tasa de satisfacción entre nuestras usuarias (del 92\%); sus pocos importantes efectos colaterales (alteración del ciclo menstrual, cefalea, mareo, acné, variaciones de peso y caída del cabello) que, aunque se presentaron en una frecuencia del $62 \%$ en algún momento de todo el período de seguimiento, aislados o simultáneamente, sólo fueron motivo de retiro en el $4,5 \%$ de los casos.

El Norplant se acompañó de una alta tasa de continuidad a 2 años $(93,2 \%)$ que dista bastante de otros métodos anticonceptivos disponibles para este grupo etario que no desea exponerse a un aborto, un embarazo de alto riesgo o no deseado. Sin embargo, un seguimiento más prolongado a 5 años será necesario para corroborar estos hallazgos.

Tabla 2

EFECTOS COLATERALES

\begin{tabular}{|lrr|}
\hline ALTERACIONES DEL CICLO & $\mathrm{n}$ & $\%$ \\
$\quad$ Manchado & 16 & 9,3 \\
$\quad$ Sangrado & 37 & 21,4 \\
$\quad$ Amenorrea & 26 & 19,3 \\
CEFALEA & 8 & 4,3 \\
MAREOS & 11 & 6,4 \\
VARIACIONES DE PESO & 8 & 4,3 \\
OTRAS & 2 & 3,4 \\
\hline
\end{tabular}

Tabla 3

SATISFACCION CON EL METODO

\begin{tabular}{|crr|}
\hline SATISFECHA & $\mathrm{n}$ & $\%$ \\
$\mathrm{Si}$ & 159 & 91,4 \\
No & 15 & 8,6 \\
DESEA RETIRO & $\mathrm{n}$ & $\%$ \\
Si & 12 & 6,8 \\
No & 162 & 93,2 \\
\hline
\end{tabular}

Tabla 4

MOTIVO DE RETIRO

\begin{tabular}{|lll|}
\hline & $\mathrm{n}$ & $\%$ \\
Alteraciones menstruales & 3 & 1,7 \\
Cefalea & 2 & 1,1 \\
Cambio de peso & 3 & 1,7 \\
Desea embarazo & 4 & 2,3 \\
\hline
\end{tabular}

\section{BIBLIOGRAFIA}

1. International Development Research Center of Canada. Choice and Challenge. Global team work in developing a contraceptive implant. 1990.

2. Díaz S., Pavez M., Miranda P., Robertson DN., Sivin I., Croxatto HB A five year clinical trial of levonorgestrel silastic implants (Norplant). Contraception 1982; 25: 447-456.

3. Croxxatto HB., Díaz S., Miranda P., Elamson K., Johansson EDB. Plasma levels of levonorgestrel in women during longterm use of Norplant. Contraception 1981; 23: 197-209.
4. Segal SJ., Alvarez-Sánchez F., Brache V., Faundes A., Vilja P., Tuohima P. Norplant implants: the mechanism of contraceptive action. Fert Steril 1991; 56: 273-277.

5. Dean M. Moutos. Levonorgestrel Subdermal Implants: Norplant. Postgrad Obst and Gyn 1993; 13(3): 1-7.

6. Singh K., Viegas OA., Patman SS. Menstrual Changes during Norplant use and return to fertility following Norplant implant removal: the Singapore experience. Advances in contraceptive delivery sistems. 1991; 7(2): 139-145. 